\title{
Design for Postplacement Mousing based on GSM in Long-Distance
}

\author{
Xin Gao*1, Bo Zhang ${ }^{2}$, Shixiong Liang ${ }^{3}$, Dong Xing ${ }^{4}$, Junlong Wang ${ }^{5}$ \\ ${ }^{1,2}$ EHF Key Laboratory of Fundamental Science, University of Electronic and Science Technology of China \\ (UESTC), Chengdu, China \\ ${ }^{3,4,5}$ National Key Laboratory of Application Specific Integrated Circuits, Hebei Semiconductor Research \\ Institute Shijiazhuang, China \\ e-mail: gaoxin_fighting@163.com, bozhang@uestc.edu.cn
}

\begin{abstract}
This design for mousing is made up of power control module, infrared sensor module, signal processing module, distance information transportation based on GSM and device of power grid. The design consists of two sets of conductors, separately linked by fire wire and null line and distributing alternatively. The major innovation is infrared sensor module with Fresnel lens, and that the infrared detecting area should be spread in one direction at least. When the mouse get into the infrared detecting area, the sensor signal of infrared detecting device is sent to power control module through signal element and then starts the device of power grid to power up to make the mouse be shocked or die. GSM module is adopted to tell that the mouse is caught successfully. This design can be placed in any position that the mouse is always out and no need of baits.
\end{abstract}

Keywords: infrared sensor module, fresnel lens, device of power grid, GSM

\section{Introduction}

Rat-problem in people's life has been existing all the time. Compared with the electric mousetrap that already exists on the market for killing the mice, this design is more practical and reliable. Remote post type mice- catching-machine based on GSM includes power supply control module, infrared sensor module, signal processing module, distance information transportation based on GSM module and power grid device. The design is simple and easy to use with low power consumption, high reliability and other advantages.

Electric mousetrap emphasis on the high voltage touching the mice. The conductors are arranged alternately that are connected by two power electrodes of the high voltage conductive circuit. The conductive circuit with high electric current can make mice die for electric shock. In general, the electrified wire netting with high voltage is without electricity, which can save electric energy and permit mice going to the electrified wire netting. The switch of the electrified wire netting giving a high voltage is located on the top of the wire netting, which is an electric infrared sensor. This infrared sensor can release infrared ray that can detect mice. And the innovation point of this design is that the sensor only detects the $1 / 3$ middle part of the electrified wire netting, and the other $1 / 3$ parts can't be detected. Once the mouse comes into the $1 / 3$ middle part of the wire netting, the electric infrared sensor device can sense the targets (namely mice). The signal of electric infrared sensor is sent to the module of power controlling through module of signal processing. At this time, the other $1 / 3$ parts are added high voltage, and if the mouse wants to leave or go further, it will be flashed or be electrocuted depending on the voltage value. In case of people touching the electricity carelessly, the device will flash the red light to remind people. There is another innovation, the infrared sensor is based on Fresnel, which will produce the signal to control the voltage when the mouse moves. Or else, there is no signal output and the electric network can't produce the power. This kind of design can decrease the electricity consumption, and won't forbid the following mice (As I mentioned above, when mice get to the middle part of the device, the Fresnel starts to work).

\section{Theory of This Design}

This design for postpositive mousing based on GSM in long-distance consists power control module, infrared sensor module, signal processing module, distance information 
transportation. The structure of the device is the place will flash mice. The most important module is the infrared sensor part which realize the function of detection, switch controlling, and power controlling. And the module of GSM completes the communication function that will send the capture situation to the specific phone. The whole device is made up of conducting bars that are connected by the power's two poles and are arranged alternatively. This kind of design results in making the conducting path including the mouse.

Figure 1 is the power grid structure of the device. In the Figure, the 5 part is the structure of the electric grid that is composed by conductors connected by the two power poles. And the voltage circulation of the device is $220 \mathrm{~V}$. The conductors are arranged alternatively and are isolated from the ground. The distance between the conductors should be less than the $1 / 2$ length of the mouse. As shown in the Figure, the support (3part) holds the Fresnel lens at the bottom of the infrared sensor (PIR). Supposing area $b$ is a circle with the radius of $b$ detected by the infrared sensor, there should be another two area $a(a>=b)$ on its sides. The sensing signal from the infrared sensor is sent part 2 which represents the signal processing module. It can control the power controlling module in which there is an electric relay to control the on-off of the power controlling module.

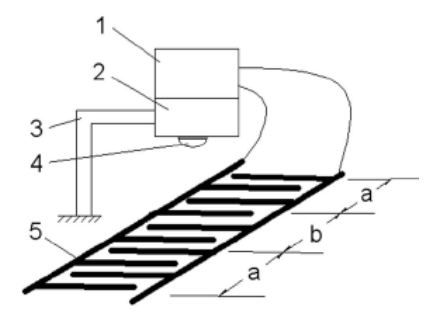

Figure1. The power grid structure of the device

Figure 2 is unit of circuit configuration of sensor signal processing. The device of the infrared sensor PIR with Fresnel lens (part 4 in the Figure) which is placed in the right front of PIR. The signal S at the output passes through C1 (filtering function) and RP1 (potential device) and then arrives at the infrared sensor processor (BISS0001 integrated circuit, shown in the Figure). When changing the distance between the infrared sensor and the electric grid, the detecting area can be changed. When adjusting RP2 (potential device), the duration of the time of the output signal can also be changed. Input signal in pin 14 shown in the Figure passes through its first operational amplifier and then export from pin 16. And the AC signal passes through capacitor C5 and then arrived at the second operational amplifier through pin 13 and the DC could not pass through C5. Then the signal exports U0 to power controlling module through pin $2[1,2,5]$. The duration time of the controlling signal $U 0$ is controlled by the delay timer composed by RP2 and C2[3,4]. If the RP2 is changed, the time of outputting the high voltage from pin 2 can be changed. The blocking timer is composed by R1 and C3, during which time the output of pin 2 will not be controlled by the input of pin14. R3, C4, R4 and C6 make up the low band pass filter that restrains high frequency signal[$[6,7,8]$.

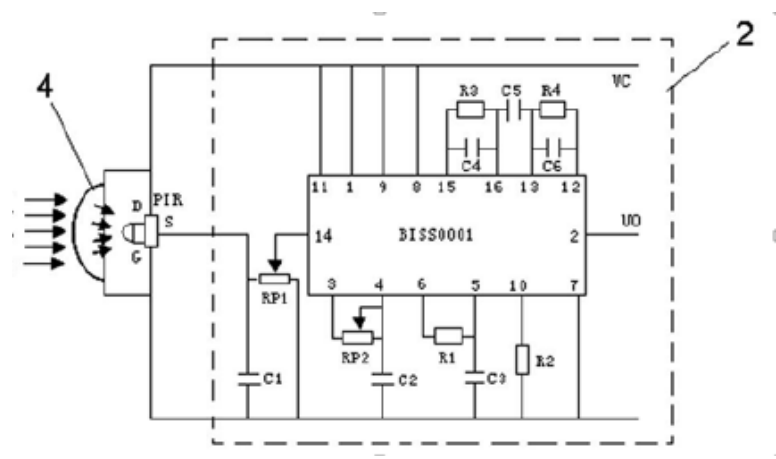

Figure 2. Circuit configuration of sensor signal processing unit

IJEEI Vol. 5, No. 3, September 2017: $259-263$ 
As shown in Figure 2, Fresnel lens (be identified by 4) is placed in the right front of the $\mathrm{PIR}$, which can cut the infrared ray radiating from the mouse to non-sequence ray and can coverage the infrared ray that can be collect by the PIR device and then be changed to electrical signal. If the mouse moves, there will be AC output, or else, there will be DC.

Figure 3 is the structure of the power control module. U0 that is output by the signal processing unit passes through R5 (resister) and $\mathrm{Q}$ (triode), which together composed the driving circuit (be identified by 1 in the Figure). And the current from the driving circuit controls the electric relay (is identified by $\mathrm{J}$ ). When there is an output signal, the contact point can be closed so that the electric grid (be identified by 5 in the Figure) can start to work. The currentlimiting resistance (R6) and the light emitting diodes (D) are both adjustable. When the electric grid are charged, D will be lighted in order to alarm people that there is electrified.

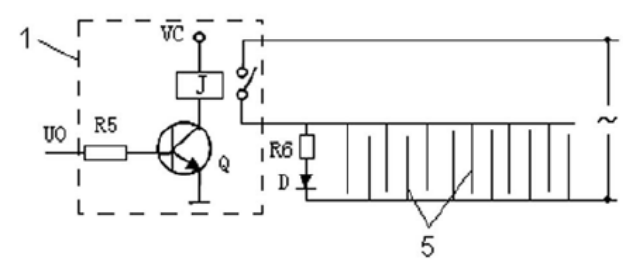

Figure 3. The structure of the power control module

In order to capture mice conveniently and deal with the mouse that has been shocked to death timely, GSM module as the communication system helps. The software refers to the serial communication between single-chip microcomputer (SCM) and GSM module, SPI communication between the host computer and slave computer, information encoding of GPS, and GSM-AT encoding. The function of the main program is to initialize GSM and send message to the phone. The function of the subprogram is to set the baud rate of the serial, start up the serial, initialize GSM module and so on. Figure 4 is the hardware base of the GSM. GSM includes radio frequency circuits and baseband to provide users with the standard AT commands, data, voice, short message and fax to provide fast, reliable, secure long-distance transmission. SIM card module shown in the Figure only accepts control from controlling module. After receiving the control signal, the control module will send information from the content to the specific mobile phone.

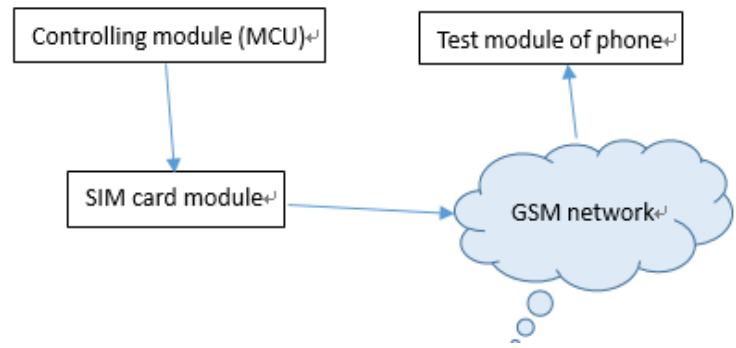

Figure 4. Hardware base of the GSM

GSM realizes the function of communication. Then there will be some introduction of the AT instructions in the following. AT instruction, instruction symbols, data, PDU data packets are transmitted in the ASCII encoding form, so it is necessary to set up mobile phone's work mode for the PDU format, referring the instruction $A T+C M G F=0[1,9,10]$. And what we should notice that end the instructions sent to mobile phone with a carriage return. For example, the instruction alignment of "AT+CMGF=0" is " $41 \mathrm{H}, 54 \mathrm{H}, 2 \mathrm{BH}, 42 \mathrm{H}, 4 \mathrm{DH}, 47 \mathrm{H}, 46 \mathrm{H}, 3 \mathrm{DH}, 30 \mathrm{H}$, $0 \mathrm{DH}$ " and the last "ODH" represents the ending of the instruction. And the flow chart of the AT instruction is shown in the following Figure 5. 


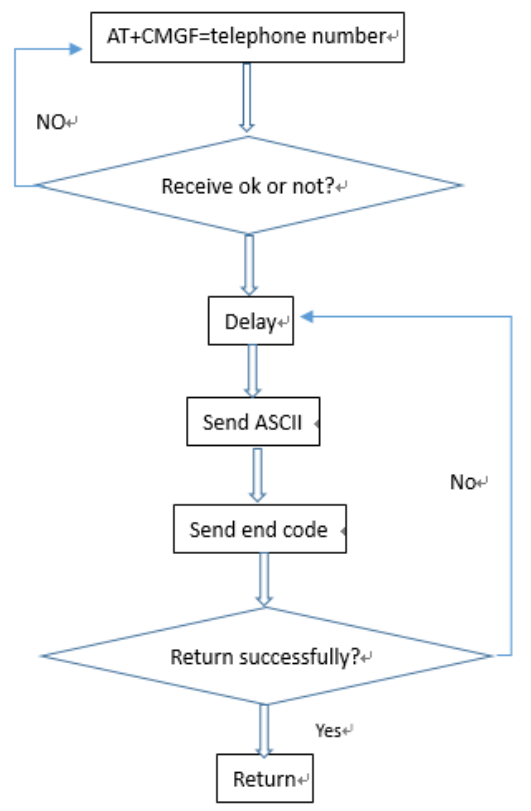

Figure 5. The flow chart of AT instruction

The pyroelectric infrared sensing device can collect signal and output AC and DC signal, which has been referred above. Then the software can change the AC and DC signal into 1 and 0 (binary system)[11]. Once the change of 0 -to- 1 is detected, in other words, the switch triger is pushed, then the power adding device powers up. When detecting the change of 1-to -0 for a period of time (can be set in the program), the powering adding device stops working. All above mean that the infrared sensoring signal can control the powering device through trriger signal.

\section{Results and Test}

This design has been tested for one week placed where mice always went. To acquire better results, the camera was set in the place. On one Monday night, a mouse was captured successfully and could not escape because of the specific design which has been described in the paper. And the phone will get the message 50 seconds later. To test the continuous capture of the mouse, the first mouse captured was not cleaned out. And the second mouse was captured on that week's Thursday. According to the result of the test, the design is potential. If the design is changed smaller and improved further, it can be applied in capturing the cockroaches.

\section{Conclusion}

This design combines mousing catcher and GSM module, and is controlled by MCU to realize the tele-control. The design based on GSM make it accessible of the communication between people and the device. And the specific design of the postplacement is one of the innovation points, that is, only the mouse goes into the electric grid, the grid can be live with electricity which can decrease the power consumption. Considering the safety, for there is only the middle $1 / 3$ part of the electric grid can be chargeable with just $110 \mathrm{~V}$ or $220 \mathrm{~V}$ and there will be a red light to remind people, so it is relatively safe.

\section{Acknowledgment}

This work is supported by the National Natural Science Foundation of China under Grant No.61301051 and supported by the Fundamental Research Funds for the Central Universities under Grant No.ZYGX2015J017.

IJEEI Vol. 5, No. 3, September 2017: $259-263$ 


\section{References}

[1] Fang Fang. Fundamentals of Mono-chip Computers Applications. Beijing: Tsinghua University Press. 2007.

[2] R Pandya. Mobile and Personal Communication Services and system. IEEE Press. 2000.

[3] GSM AT instructions handbook. Nanjing. Nanjing Aoyi electronics Co.,Ltd.

[4] Jincun Yu. Principle and Interface Technology. Xian: Xdian University Press. 2004.

[5] Tiyun Guo. Mobile Communication [M]. Xian: Xdian University Press. 2003.

[6] Zhanyou Sha. Design of Peripheral Circuit of Single Chip Microcomputer. Beijing: Electronic Industry Publishing House. 2003.

[7] A Zafft, E Agu. Malicious WiFi Networks: A First Look. 7th IEEE Workshop on Security in Communication Networks. 2012.

[8] CD Oancea. GSM infrastructure used for data transmission. 2011 7th International Symposium on Advanced Topics in Electrical Engineering. 2011.

[9] HF Qi, XH Yang, R Jiang, B Liang, SJ Zhou. Novel End-to-End Voice Encryption Method in GSM System. Proceedings of 2008 IEEE International Conference on Networking, Sensing and Control. 2008.

[10] F Bode. Introduction \& Update to an open standard for instrument control: SCPI standard commands for programmable instruments. the Wescon '92, Anaheim, CA, USA. 1992.

[11] Z Zhu, H Zhao, L Shen. The application of structure arrays and files in the SCPI parsing system. 2010 International Conference on Intelligent Computation Technology and Automation. 2010. 